\title{
A Textile Platform Using Continuous Aligned and Textured Composite Microfibers to Engineer Tendon-to-Bone Interface Gradient Scaffolds
}

\author{
Isabel Calejo, Raquel Costa-Almeida, Rui L. Reis, and Manuela E. Gomes*
}

Tendon-to-bone interfaces exhibit a hierarchical multitissue transition. To replicate the progression from mineralized to nonmineralized tissue, a novel 3D fibrous scaffold is fabricated with spatial control over mineral distribution and cellular alignment. For this purpose, wet-spun continuous microfibers are produced using polycaprolactone $(\mathrm{PCL}) /$ gelatin and $\mathrm{PCL} /$ gelatin/hydroxyapatite nano-to-microparticles (HAp). Higher extrusion rates result in aligned $\mathrm{PCL} /$ gelatin microfibers while, in the case of PCL/gelatin/HAp, the presence of minerals leads to a less organized structure. Biological performance using human adipose-derived stem cells (hASCs) demonstrates that topography of PCL/gelatin microfibers can induce cytoskeleton elongation, resembling native tenogenic organization. Matrix mineralization on PCL/gelatin/HAp wet-spun composite microfibers suggest the production of an osteogenic-like matrix, without external addition of osteogenic medium supplementation. As proof of concept, a 3D gradient structure is produced by assembling PCL/gelatin and PCL/gelatin/HAp microfibers, resulting in a fibrous scaffold with a continuous topographical and compositional gradient. Overall, the feasibility of wet-spinning for the generation of continuously aligned and textured microfibers is demonsrated, which can be further assembled into more complex 3D gradient structures to mimic characteristic features of tendon-to-bone interfaces.

\section{Introduction}

Tendon-to-bone failure due to trauma or severe illness is an increasing healthcare problem. Conservative treatments are often not effective or inadequate and surgical application of grafts is the gold standard approach to clinical management of such injuries. Despite recent advances in surgical techniques, interface repair still fails up to $95 \%$ of the time due to the formation of fibrotic tissue. ${ }^{[1]}$ The major problem associated with tendon-to-bone junction regeneration is the fact that, after failure, there is a disordered matrix deposition rather than the formation of organized collagen fibers, affecting tendon tissue integration and leading to a disorganized graded interface. ${ }^{[2]}$ Moreover, the gradual loss of mineral content is also believed to contribute to the observed mechanically inferior interface, resulting in tissue rupture. ${ }^{[3]}$ Therefore, tissue-engineered approaches combining biological and engineering sciences are essential to regenerate these complex interfaces. In this regard, interfacial tissue engineering is a fascinating research field with sub-

I. Calejo, Dr. R. Costa-Almeida, Prof. R. L. Reis, Prof. M. E. Gomes 3B's Research Group

i3Bs - Research Institute on Biomaterials

Biodegradables and Biomimetics

University of Minho

Headquarters of the European Institute of Excellence on Tissue

Engineering and Regenerative Medicine

AvePark, Parque de Ciência e Tecnologia

Zona Industrial da Gandra, 4805-017 Barco, Guimarães, Portugal

E-mail:megomes@i3bs.uminho.pt

I. Calejo, Dr. R. Costa-Almeida, Prof. R. L. Reis, Prof. M. E. Gomes ICVS/3B's- PT Government Associate Laboratory

4806-909 Braga, Guimarães, Portugal

Prof. R. L. Reis, Prof. M. E. Gomes

The Discoveries Centre for Regenerative and Precision Medicine

Headquarters at University of Minho

Avepark, 4805-017 Barco, Guimarães, Portugal

The ORCID identification number(s) for the author(s) of this article can be found under https://doi.org/10.1002/adhm.201900200.

DOI: 10.1002/adhm.201900200 stantial focus on the development of strategies to emulate complex and multiphasic tissue interfaces, such as the one interconnecting tendon to bone. Particularly, providing the necessary biological cues in one single unit, while recreating the mechanical requirements of the native tissue would be of great importance.

Several approaches are available for the fabrication of naturally derived or synthetic fibers for tissue engineering applications, including electrospinning, meltspinning, interfacial complexation, or microfluidic spinning. ${ }^{[4]}$ However, these techniques present some limitations as the use of highly toxic organic solvents, voltages, and temperatures known to partially denature the structure of natural polymers, ${ }^{[5]}$ lack of control of scaffold porosity involving the use of other techniques to obtain larger pores to enable cell infiltration ${ }^{[6]}$ and the impossibility to scale-up the fiber production limiting the application to large lab-scale systems. ${ }^{[4]}$ Strikingly, among available fiber processing techniques, wet-spinning appears as a powerful tool to convert biomolecules into fibers without the need of high voltages or 
temperatures and likely less denaturation. ${ }^{[7,8]}$ This manufacturing process has been present in textile industry for many years and is based on a nonsolvent-induced phase inversion, which involves the extrusion of a polymeric solution directly into a coagulation bath. ${ }^{[8]}$ This bath is normally composed of a poor solvent (nonsolvent) or a nonsolvent/solvent mixture having in mind the processed polymer to form a coagulating filament, that will solidify as a continuous polymeric fiber. ${ }^{[9,10]}$ Compared to other available techniques, wet-spinning presents several advantages such as low cost, high yield, adjustable fibers diameter and, ultimately scaffolds porosity, and mild production conditions. ${ }^{[5,10]}$ Furthermore, fibers are very easy to handle and assemble by textile techniques. ${ }^{[9,10]}$ Therefore, wetspun fibers have been used to produce different tissue analogs such as cartilage, ${ }^{[11]}$ bone, ${ }^{[12]}$ and even neural tissue. ${ }^{[13]}$

Herein, we aimed at developing a gradient scaffold by assembling microfibers with distinct topographical and compositional features tailored to guide cell alignment and simultaneously match the mechanical properties of the native tissue. Wet-spinning was used to produce two types of microfibers toward mimicking the soft (tendon) and hard (bone) tissues constituting the tendon-to-bone interface. For this purpose, synthetic/natural origin blend formulations of polycaprolactone and gelatin (PCL/gelatin) and PCL/gelatin incorporating nano-to-microsized hydroxyapatite (HAp) particles were used. Different flow rates were tested to assess their effect on wet-spun fibers diameter and mechanical properties. The biological performance of the developed microfibers was assessed by culturing human adiposederived stem cells (hASCs) onto the fibers for a period of 14 days. As proof of concept, knitting (crochet) was used to create a 3D fibrous scaffold using the produced fibers to generate a mineral gradient within the developed structure. Also, the formation of specific regions was assessed by studying the deposition of characteristic collagen matrices by hASCs cultured onto scaffolds.

\section{Results}

\subsection{Fabrication and Morphological Characterization of Wet-Spun Composite Microfibers}

Wet-spun fiber production set-up is shown in Figure 1A. Two different polymeric solutions were injected into a supporting coagulation bath. While the solutions were injected, fibers were instantaneously being formed while a roller was collecting them at a constant speed. This fabrication set-up allowed obtaining a continuous thread (Video S1, Supporting Information) that could be easily manipulated and stored (Figure 1A). The initial polymeric solutions were designed to mimic the collagenous composition of tendon (gelatin) and the mineral composition of bone (HAp), along with the topography of both tissues. In order to evaluate the effect of collection speed on the wet-spun fibers diameter, as well as, alignment, three different flow rates $\left(1,0.5\right.$, and $0.25 \mathrm{~mL} \mathrm{~h}^{-1}$ ) were tested (Figure $1 \mathrm{~B}$ ). By varying the flow rates, wet-spun fibers could be obtained with diameters in the range of $110-400 \mu \mathrm{m}$. The highest extraction rate $\left(1 \mathrm{~mL} \mathrm{~h}^{-1}\right)$ resulted in significantly thicker fibers $(368.25 \pm 29.47 \mu \mathrm{m}$ for PCL/gelatin and $332.99 \pm 41.85 \mu \mathrm{m}$ for PCL/gelatin/HAp) compared with lower flow rates of 0.5 and $0.25 \mathrm{~mL} \mathrm{~h}^{-1}$
( $p<0.0001$, Figure $1 \mathrm{C}$-i,ii). The lowest flow rate resulted in significantly thinner fibers $(p<0.0001,126.32 \pm 7.87 \mu \mathrm{m}$ for PCL/gelatin and $182.50 \pm 22.22 \mu \mathrm{m}$ for PCL/gelatin/HAp). Interestingly, the conjugation of HAp in the polymeric solution and lower extrusion rates led to the production of fibers with higher diameters (Table S1, Supporting Information). Furthermore, HAp incorporation also led to different topographical orientation (Figure 1D). In the directionality graphs, an absence of a peak means a random orientation of fibers, as observed in the case of PCL/gelatin/HAp fibers, while the presence of a sharp peak in the orientation angle frequency represents a tendency to align in a specific orientation. Strikingly, a flow rate of $1 \mathrm{~mL} \mathrm{~h}^{-1}$, seemed to induce a higher anisotropic alignment in PCL/gelatin fibers, while in contrast fiber alignment was less evident in the case of PCL/gelatin/HAp fibers.

Additionally, as observed in Figure 1, PCL/gelatin/HAp fibers were characterized by the presence of pores due to the incorporation of differently sized HAp particles. Interestingly, a flow rate of $1 \mathrm{~mL} \mathrm{~h}^{-1}$ leads to the accumulation of larger particles in fibers $\left(0.5 \mathrm{~mL} \mathrm{~h}^{-1}, p<0.008 ; 0.25 \mathrm{~mL} \mathrm{~h}^{-1}, p<0.0009\right.$; Figure S1A-i in the Supporting Information), while no significant differences were found between particles size on fibers produced at lower flow rates. Therefore, a significantly higher pore volume was found in the highest flow rate in comparison with the lowest $\left(0.25 \mathrm{~mL} \mathrm{~h}^{-1}, p<0,02\right.$, Figure S1A-ii in the Supporting Information). Similarly, lowering the flow rate, resulted in a decrease of porosity amount. When addressing the interconnectivity of these fibers, it was observed a significantly higher interconnection of pores in flow rates of 0.5 and $0.25 \mathrm{~mL} \mathrm{~h}^{-1}$ in comparing with $1 \mathrm{~mL} \mathrm{~h}^{-1}(p<0.008$, Figure S1A-iv in the Supporting Information).

\subsection{Chemical and Mechanical Characterization}

The characterization of the functional groups of PCL, gelatin, hydroxyapatite, PCL/gelatin and PCL/gelatin/HAp was performed by FTIR. Infrared spectra of raw materials and developed fibers are shown in Figure 2A-i,ii. In both PCL/ gelatin and PCL/gelatin/HAp several PCL characteristic bands are observed in 2931 (asymmetric stretching $-\mathrm{CH}_{2}$ ), 2858 (symmetric stretching $-\mathrm{CH}_{2}$ ), $1292(\mathrm{C}-\mathrm{O}$ and $\mathrm{C}-\mathrm{C}$ stretching) and 1239 (C-O-C, asymmetric stretching). The characteristic bands for gelatin were also found in 3433 (N-H stretching, amide bond), 1653 ( $\mathrm{C}=\mathrm{O}$ stretching, amide I) and $1543 \mathrm{~cm}^{-1}$ (N-H bending, amide II), being also observed in both fibers. Additionally, HAp particles presented one critical infrared band located in $1041 \mathrm{~cm}^{-1}$ corresponding to the group of $v 3 \mathrm{PO} 4$ which appeared for PCL/gelatin/HAp fibers (Figure 2A-ii).

Concerning the mechanical performance of composite fibers, tensile properties of PCL/gelatin and PCL/gelatin/HAp are summarized in Figure $2 \mathrm{~B}$ and Table 1 . The mechanical properties were examined in terms of young's modulus, yield strength, yield strain, and strain to failure. Different blend formulations and tested flow rates resulted in a significant impact on the Young's modulus of produced wet-spun fibers. Regarding PCL/gelatin fibers, the highest extrusion flow rate $\left(1 \mathrm{~mL} \mathrm{~h}^{-1}\right)$ resulted in fibers with significantly lower Young's modulus $(115.30 \pm 16.33 \mathrm{MPa}, p<0.0001)$ in comparison with fibers 
A
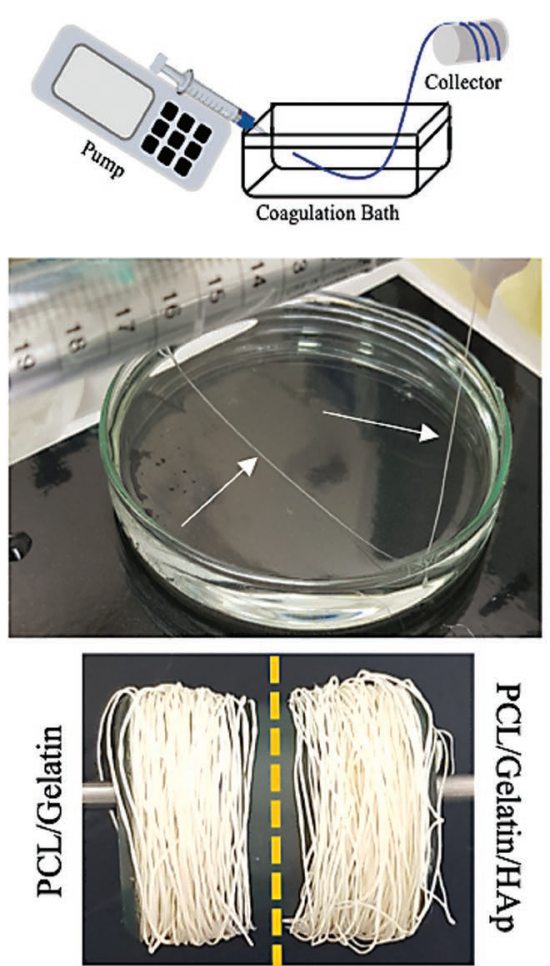

D

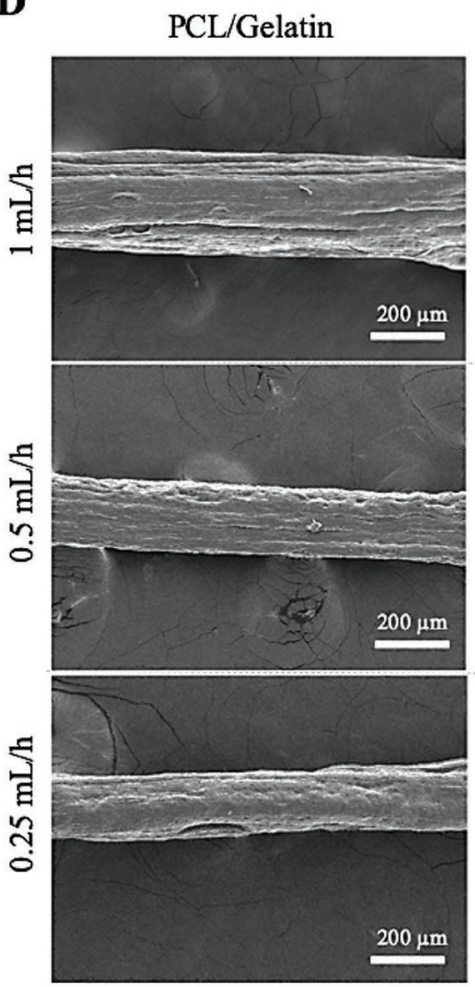

B

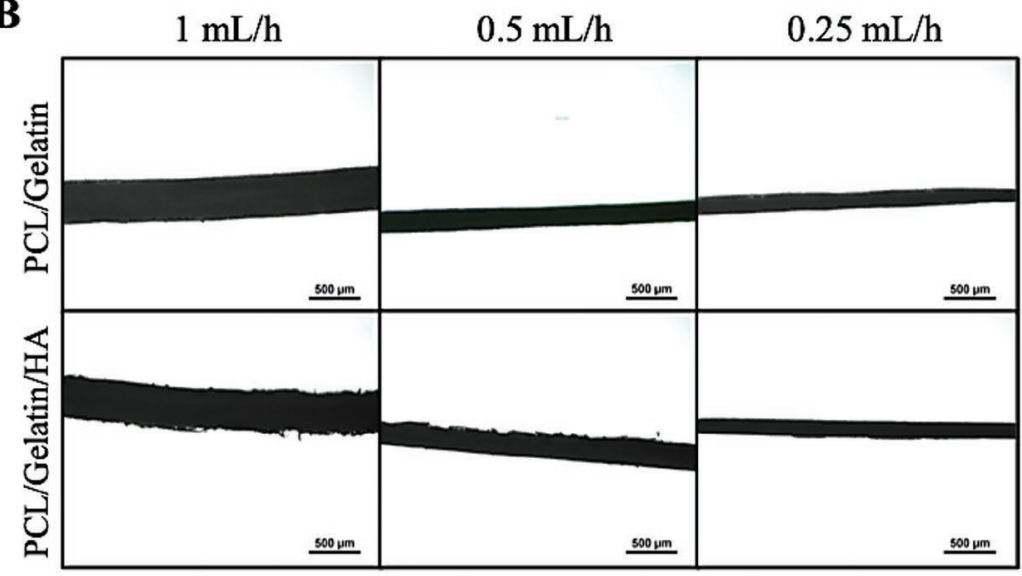

C

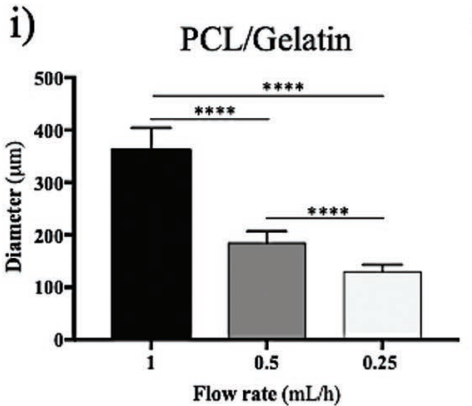

ii)

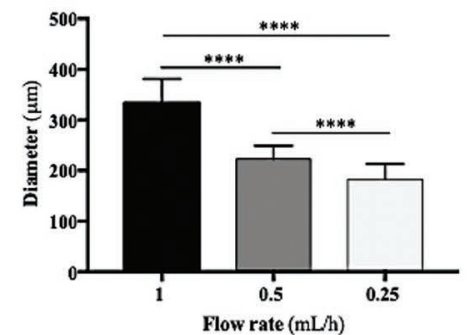

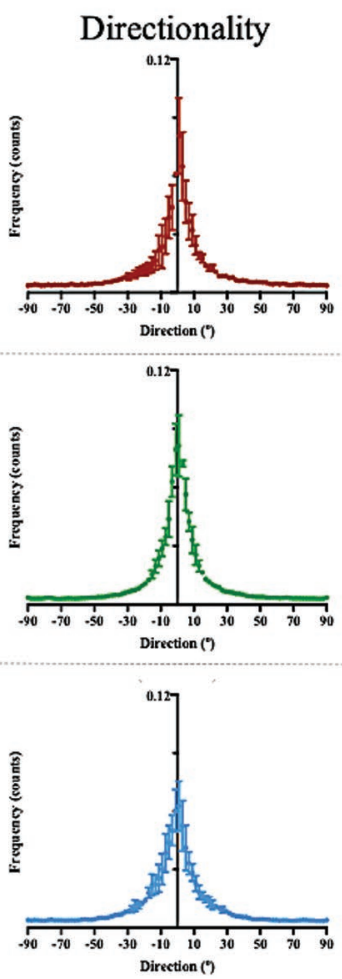
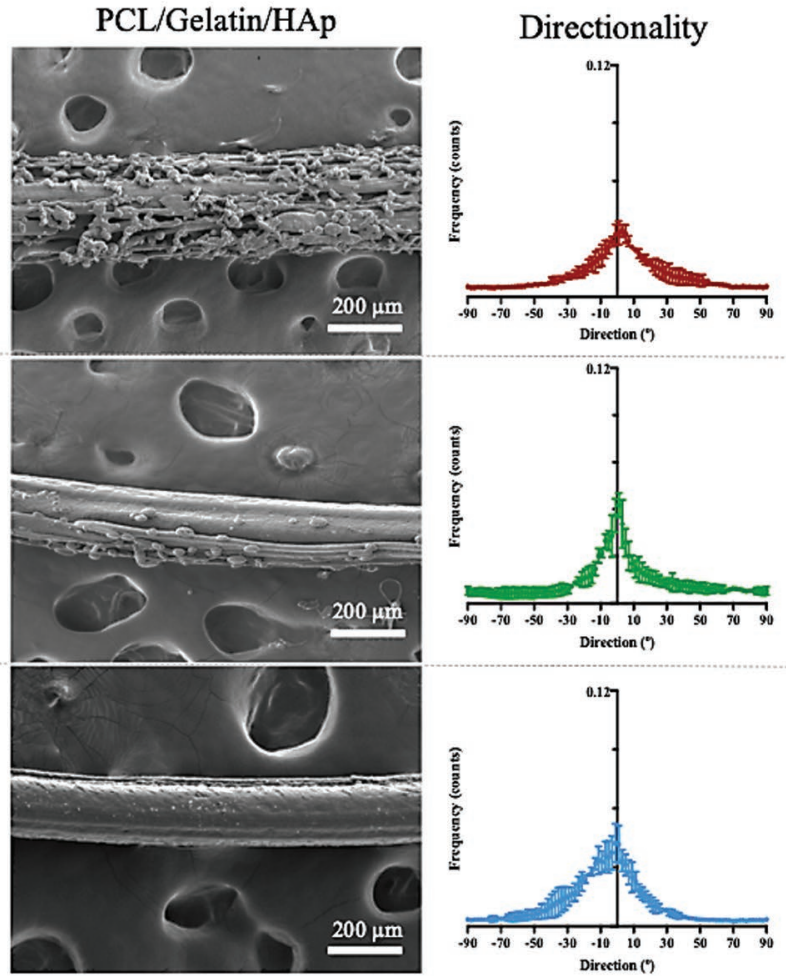

Figure 1. Schematic representation of wet-spinning setup and morphology of PCL/gelatin and PCL/gelatin/HAp wet-spun fibers. A) Continuous wet-spinning system. Arrows point fibers formation. Wet-spun fibers can be easily manipulated and collected in rollers for further use. B) Optical microscopy images of produced wet-spun fibers according to the different formulations and flow rates. Scale bars, $500 \mu \mathrm{m}$. C) Average diameter of the produced (i) PCL/Gelatin $(n=6)$ and (ii) PCL/gelatin/HAp fibers $(n=6)$ ( for the different fibers and the topographical directionality was assessed, as observed by the frequency counts represented in the graphs in the left. 
A

i)

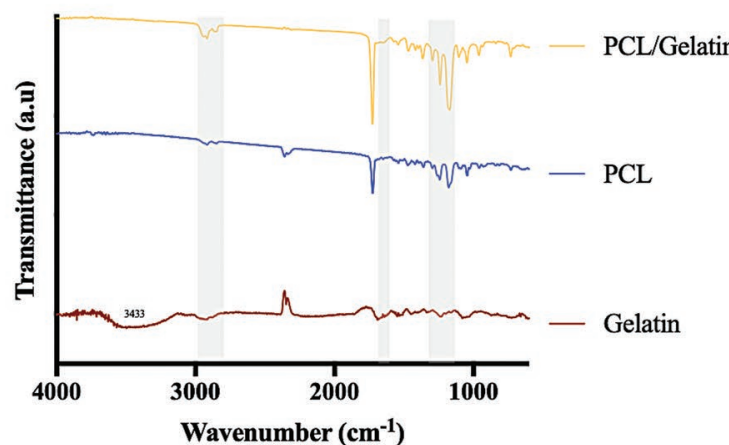

B

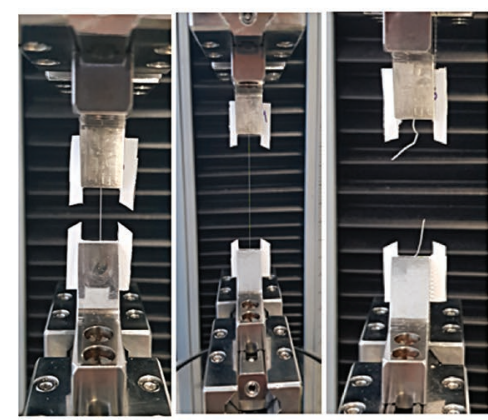

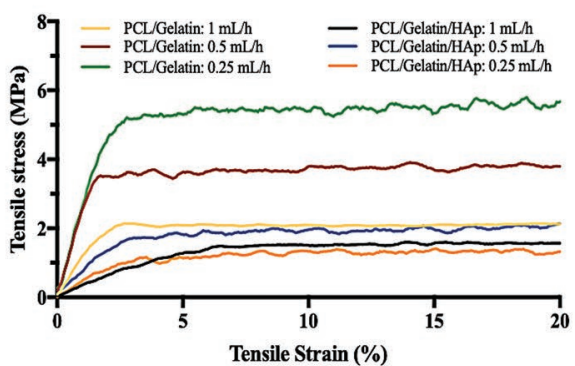

ii)

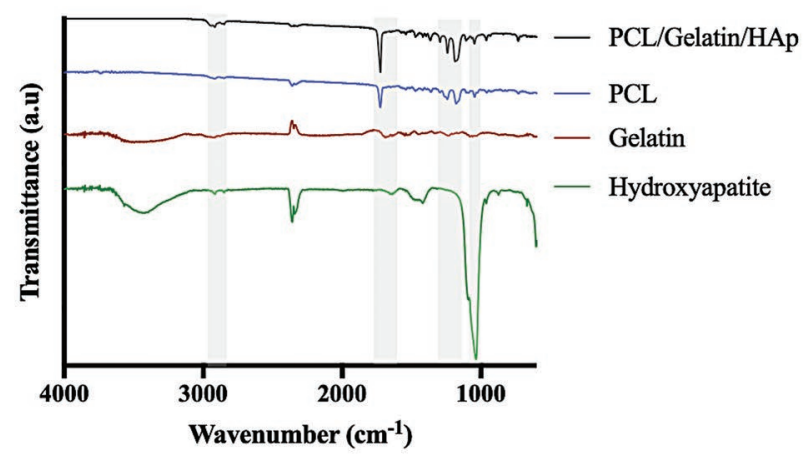

iii)

Figure 2. Chemical analysis and mechanical properties of PCL/gelatin and PCL/gelatin/HAp wet-spun fibers. A) FTIR spectra of neat PCL, gelatin and hydroxyapatite, as well as (i) PCL/gelatin and (ii) PCL/gelatin/HAp wet-spun fibers with bands of interest identified. B) Mechanical testing of specimens. (i) The samples were placed between the tester grips and the tensile tests were performed until break point. (ii) Characteristic tensile stress-tensile strain curves of the distinct formulations and respective flow rates were obtained and (iii) the young's modulus calculated. Statistically significant differences between tested flow rates in PCL/gelatin fibers production in comparison with the same tested conditions in PCL/gelatin/HAp fibers are

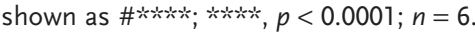

produced at lower flow rates $\left(0.5 \mathrm{~mL} \mathrm{~h}^{-1}, 251.80 \pm 25.51 \mathrm{MPa}\right.$; $0.25 \mathrm{~mL} \mathrm{~h}^{-1}, 241.10 \pm 41.92 \mathrm{MPa}$, Figure 2B-iii). An opposite tendency was observed for PCL/gelatin/HAp fibers where Young's modulus ranged from $35.39 \pm 6.19$ to $59.13 \pm 7.87 \mathrm{MPa}$ (Figure 2B-iii), without significant differences between Young's moduli of fibers obtained from different flow rates. Additionally, results indicated that the addition of HAp particles caused a decrease in the tensile properties of composite fibers, in comparison to PCL/gelatin fibers, as observed by the lower young's modulus (Figure 2B-ii,iii, Table 1). Interestingly, fibers produced at lower extrusion flow rates present higher plasticity, having higher strain-to-failure. In contrast, fibers produced at $1 \mathrm{~mL} \mathrm{~h}^{-1}$ are more elastic, exhibiting higher yield strain and strain to failure (Table 1).

Table 1. Mechanical properties of wet-spun fibers produced at different flow rates.

\begin{tabular}{lcccc}
\hline Specimen & & $\begin{array}{c}\text { Yield strength } \\
{[\mathrm{MPa}]}\end{array}$ & $\begin{array}{c}\text { Yield strain } \\
{[\%]}\end{array}$ & $\begin{array}{c}\text { Strain to failure } \\
{[\%]}\end{array}$ \\
\hline PCL/gelatin & $1 \mathrm{~mL} \mathrm{~h}^{-1}$ & $2.12 \pm 0.38$ & $2.02 \pm 0.28$ & $164.45 \pm 60.41$ \\
& $0.5 \mathrm{~mL} \mathrm{~h}^{-1}$ & $4.67 \pm 1.29$ & $1.93 \pm 0.42$ & $295.08 \pm 95.04$ \\
& $0.25 \mathrm{~mL} \mathrm{~h}^{-1}$ & $3.69 \pm 0.70$ & $1.63 \pm 0.21$ & $476.15 \pm 224.00$ \\
PCL/gelatin/HAp & $1 \mathrm{~mL} \mathrm{~h}^{-1}$ & $0.61 \pm 0.12$ & $2.10 \pm 0.32$ & $360.77 \pm 69.13$ \\
& $0.5 \mathrm{~mL} \mathrm{~h}^{-1}$ & $1.05 \pm 0.21$ & $1.81 \pm 0.24$ & $442.59 \pm 143.12$ \\
& $0.25 \mathrm{~mL} \mathrm{~h}^{-1}$ & $0.72 \pm 0.17$ & $2.33 \pm 1.46$ & $492.31 \pm 264.48$ \\
\hline
\end{tabular}

\subsection{Biological Performance of Wet-Spun Composite Microfibers}

Human adipose-derived stem cells were used to study the ability of PCL/gelatin and PCL/gelatin/HAp wet-spun fibers to provide the appropriate microstructural and topographical cues to support a tenogenic-like cellular organization or osteogenic-like matrix compositional features, respectively. For this reason, fibers produced at a flow rate of $1 \mathrm{~mL} \mathrm{~h}^{-1}$ were chosen for biological studies with seeded cells for a culture period of 14 days in basal medium. The rationale for selecting this fabrication condition relies on the aforementioned topographical and structural features of obtained microfibers. On the one hand, the anisotropic alignment of PCL/gelatin wet-spun composite microfibers extruded at a rate of $1 \mathrm{~mL} \mathrm{~h}^{-1}$ was hypothesized to support cell alignment, replicating tendon cell organization. On the other hand, the compositional and isotropic features of PCL/gelatin/HAp were envisioned to trigger an osteogenic behavior on seeded hASCs.

\subsubsection{Cell Viability and Morphology of hASC-Seeded Microfibers}

After seeding of hASCs, microfibers were visually inspected daily under an optical microscope to monitor cell distribution and organization along the fibers. A well-distributed layer of cells and new matrix enveloping the microfibers could be observed after 7 days of culture (Figure 3A-i,B). This initial 
A

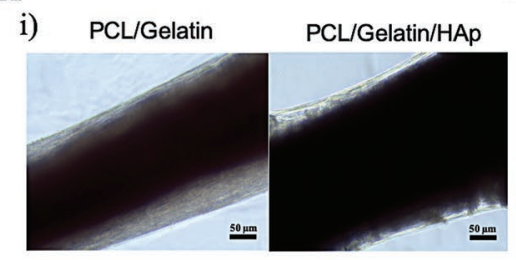

ii)

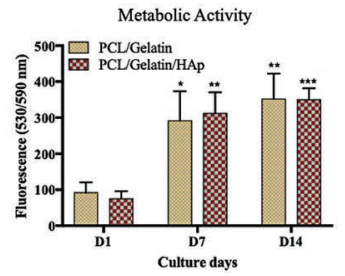

D

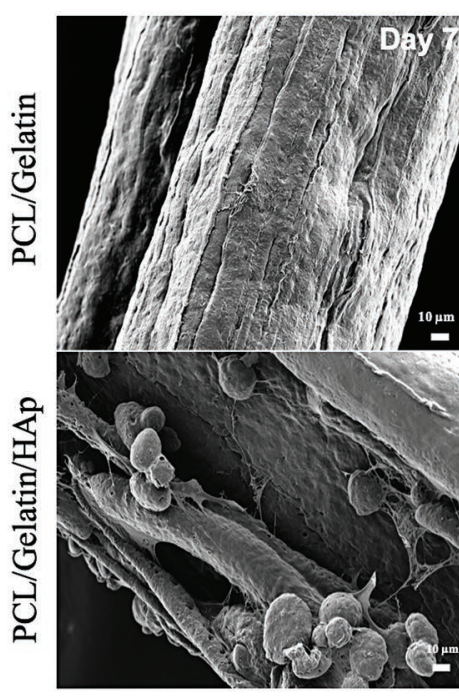

B

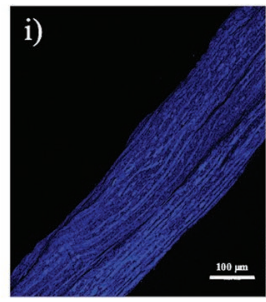

iii)

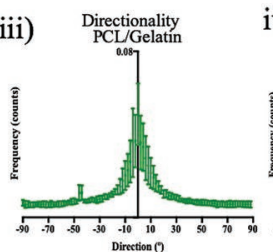

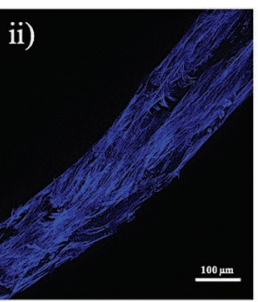

iv) $\begin{gathered}\text { Directionality } \\ \text { PCL/Gelatin/HAp }\end{gathered}$

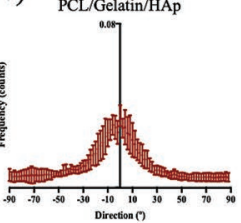

C

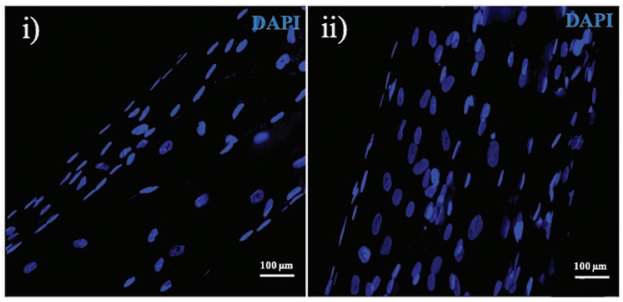

iii)

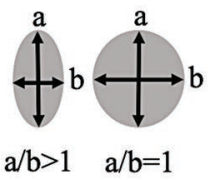

Nuclei Elongation

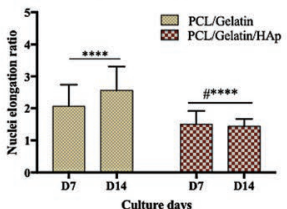

$\mathbf{E}$

Day 7

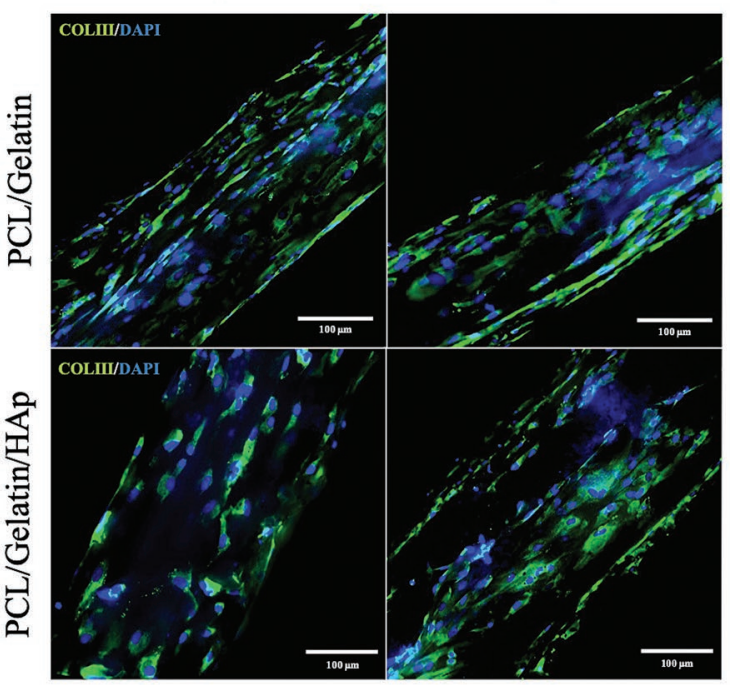

Figure 3. Cell activity, morphometric analysis of hASCs and ECM organization in PCL/gelatin and PCL/gelatin/HAp wet-spun composite microfibers produced at $1 \mathrm{~mL} \mathrm{~h}$-1. A) (i) Optical microscopy images of hASC-seeded microfibers after 7 days of culture in basal medium condition. Scale bars, $50 \mu \mathrm{m}$. (ii) Metabolic activity of seeded hASCs during 14 days of culture, determined by Alamar blue assay. Statistically significant differences are shown as $*, p<0.03, * *, p<0.001$, ***, $p<0.0003 ; n=4$. B) Confocal images of actin filaments of hASCs (phalloidin, blue) and directionality of actin filaments orientation angle in (i,iii) PCL/gelatin $(n=3)$ and (ii,iv) PCL/gelatin/HAp $(n=3)$ after 14 days of culture. Scale bars, $100 \mu$ m. C) Fluorescence microscopy images of cell nuclei (DAPI, blue) on (i) PCL/gelatin and (ii) PCL/gelatin/HAp wet-spun composite microfibers after 14 days of culture. Scale bars, $100 \mu \mathrm{m}$. (iii) Nuclei aspect ratio of hASCs cultured on both types of microfibers. Statistically significant differences

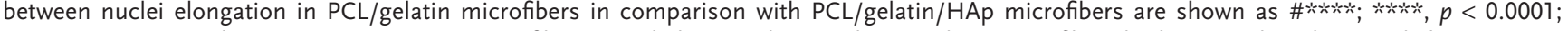
$n=3$. D) Scanning electron microscope images of hASC-seeded PCL/gelatin and PCL/gelatin/HAp fibers both at 7 and 14 days. Scale bars, $10 \mu \mathrm{m}$. E) Expression of matrix proteins observed by immunolabeling against collagen type III after 7 and 14 days of culture in both microfiber formulations. Scale bars, $100 \mu \mathrm{m}$.

observation suggests that the developed PCL/gelatin and PCL/ gelatin/HAp wet-spun microfibers were able to support the adhesion, proliferation, and possible new matrix synthesis by seeded cells. Hence, cell viability was further assessed by alamar blue assay using $1 \mathrm{~cm}$ long hASC-seeded microfibers (Figure 3A-ii). A significant increase on hASCs metabolic activity was registered from day 1 to days 7 and 14 in both PCL/ gelatin (Day 7, $p<0.03$, Day 14, $p<0.001$ ) and PCL/gelatin/ HAp microfibers (Day 7, $p<0.001$, Day 14, $p<0.0001$ ). No differences were observed at the correspondent days between both fiber formulations.

\subsubsection{F-Actin Alignment and Nuclei Elongation}

Given the importance of replicating the topography of two different tissues composing tendon-to-bone junction, it was also investigated whether produced fibers would modulate hASCs alignment and nuclei elongation. For that, confocal images were acquired after 7 and 14 days of culture and both cytoskeletal alignment and nuclei aspect ratio were evaluated. In Figure 3B-i, acquired images of actin filaments of hASCs cultured for 14 days on PCL/gelatin show that cell cytoskeleton followed the topography of microfibers, as seen by the resulting 
layer of highly aligned and elongated cells and organization of actin filaments. In contrast, even though some alignment is observed in cell cytoskeleton in contact with PCL/gelatin/HAp microfibers, a layer of randomly organized/ nonaligned cells was also observed (Figure 3B-ii). This behavior was confirmed by the directionality analysis of confocal images. The layer of highly aligned hASCs observed in PCL/gelatin microfibers led to the presence of a sharper peak in a specific orientation angle frequency, representing an alignment tendency to one specific orientation (Figure 3B-iii), resembling tendon anisotropic cellular alignment; while, in contrast, a smoother peak was noticed in the case of PCL/gelatin/HAp microfibers, suggesting a more random cytoskeleton orientation (Figure 3B-iv). Additionally, the average aspect nuclei ratio of hASCs in contact with PCL/ gelatin microfibers after 7 and 14 days of culture was found to range between $2.07 \pm 0.67$ and $2.56 \pm 0.75$, respectively, which is considerably high. Moreover, hASCs tended to elongate overtime in culture (Figure 3C-i,iii, $p<0.0001$ ). In contrast, hASCs cultured on PCL/gelatin/Hap microfibers presented statistically significant lower nuclei aspect ratio (between $1.51 \pm 0.41$ at day 7 and $1.44 \pm 0.23$ at day $14 ; p<0.0001$ ), which was confirmed by the presence of more round-shaped nuclei (Figure 3C-ii).

\subsubsection{Extracellular Matrix Production and Organization}

The extracellular cellular matrix is a dynamic structure, which interacts with cells regulating important processes such as proliferation, migration, and differentiation. Therefore, to better understand the effect of both wet-spun fibers on hASCs ECM deposition and organization, SEM images of both hASCseeded microfibers were acquired. In accordance with optical microscopy findings, it was clear the existence of a layer of cells and matrix covering the surface of PCL/gelatin fibers, as well as PCL/gelatin/HAp fibers (Figure 3D) after 7 and 14 days of culture. Remarkably, the presence of HAp on the surface of PCL/gelatin/HAp fibers, which led to an increased surface roughness, enabled cells to stretch (Figure 3D-ii, arrows). Additionally, due to increased evidence of the key role of ECM components, mainly the deposition of collagen type III (COLIII) known to be involved in the development and healing process of bone ${ }^{[14]}$ and tendon/ligament, ${ }^{[15]}$ the expression of COLIII was evaluated in hASC-seeded microfibers after 7 and 14 days of culture by immunostaining. Acquired fluorescence images demonstrated that collagen type III was synthesized over time by hASCs in both PCL/Gelatin and PCL/Gelatin/HAp fibers (Figure 3E). Furthermore, the wide spread immunolabeling observed over the surface of PCL/gelatin and PCL/gelatin/ HAp fibers was indicative of a cell migration over culture time while demonstrating the ability of produced fibers to support the growth of cells within the 14 days of culture in basal conditions (Figure 3E).

\subsubsection{Matrix Mineralization}

The mineralization of ECM is regarded as an important event during osteoblast differentiation. ${ }^{[16]}$ Therefore, the ability of wet-spun composite microfibers to support bone-like extracellular matrix deposition was evaluated by AZ staining of both fibers after culturing with hASCs for 7 and 14 days in basal medium (Figure 4A). Optical images of stained fibers confirmed an absence of mineralization in PCL/gelatin microfibers, while an extensive mineral deposition was observed in PCL/gelatin/HAp microfibers, as soon as 7 days of culture in basal medium conditions. Moreover, fibers without cells were used as controls, demonstrating the presence of HAp particles in PCL/gelatin/HAp microfibers (Figure 4B). Matrix
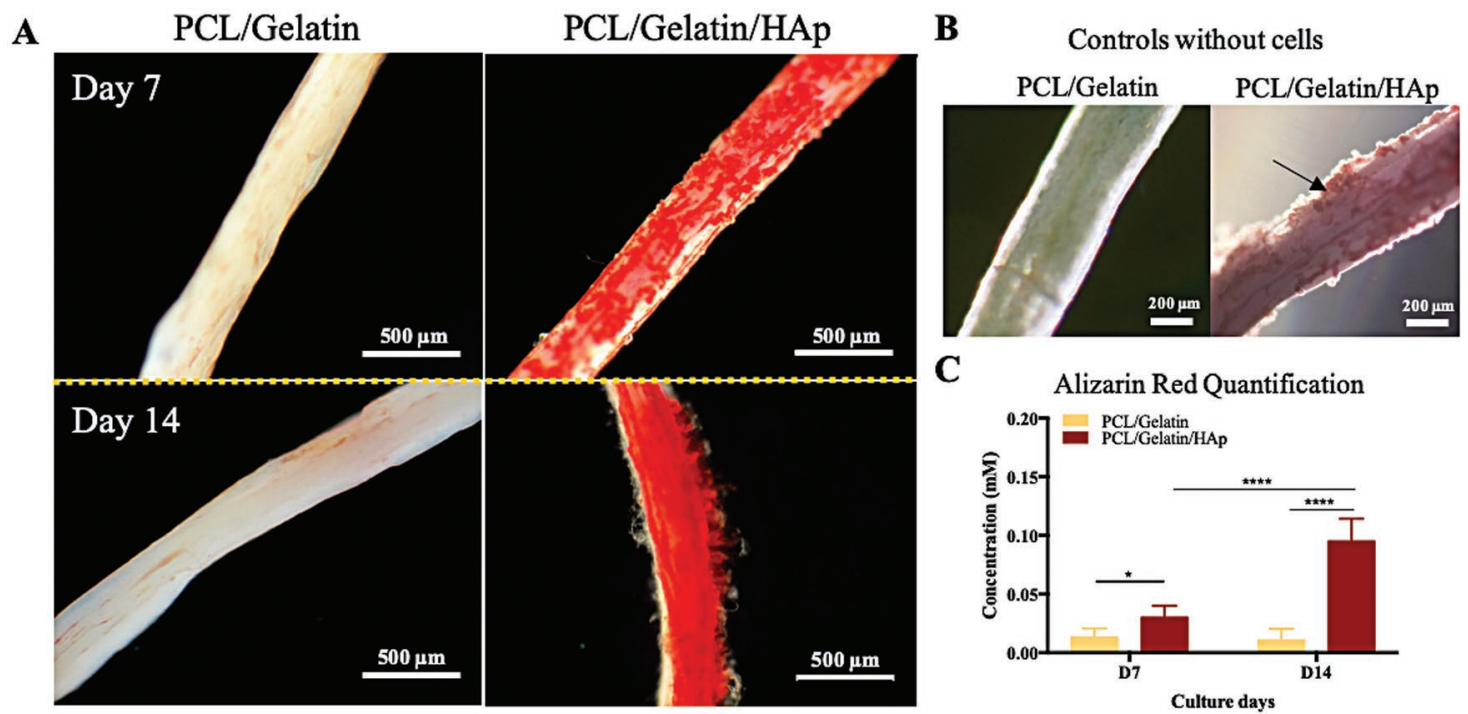

Figure 4. Alizarin red staining of $\mathrm{PCL} /$ gelatin and $\mathrm{PCL} /$ gelatin/HAp wet-spun composite microfibers. A) Matrix mineralization observed by optical microscopy after 7 and 14 days of culture of hASCs in PCL/gelatin and PCL/gelatin/HAp fibers. Scale bars, $500 \mu \mathrm{m}$. B) Fibers without cells were used as controls. The presence of HAp particles is observed in the case of PCL/gelatin/HAp fibers. Scale bars, $200 \mu \mathrm{m}$. C) Alizarin red dye quantification performed after 7 and 14 days of culture, using the cetylpyridinium method. Statistically significant differences are shown as $*, p<0.04, * * * *$, $p<0.0001, n=3$. 
mineralization in fibers was further confirmed by AZ quantification (Figure 4C). Statistically significant differences were observed between hASCs cultured in PCL/gelatin and PCL/gelatin/HAp fibers at $7(p<0.04)$ and $14 \mathrm{~d}$ of culture $(p<0.0001)$. This effect was more pronounced with time in culture in PCL/ gelatin/HAp microfibers. Indeed, a significant increase was observed in AZ quantification from day 7 to day $14(p<0.0001)$, demonstrating intensification of mineral deposition. These results are attributed to the presence of HAp, which led to increased matrix mineralization, indicating a possible differentiation of hASCs toward the osteogenic lineage, without any osteogenic-inductive medium supplementation.

\subsection{Textile Assembling of a 3D Gradient Fibrous Scaffold}

Taking advantage of the main characteristic of textile techniques which relies on the possibility to assemble fibers into bigger and adjustable constructs while preserving the characteristics of the single units, ${ }^{[4]}$ produced fibers were incrementally assembled by knitting (crochet) aiming at the generation of a 3D fibrous structure with a compositional and topographical gradient to mimic the tendon-to-bone junction. Macroscopic images show a fibrous scaffold where PCL/gelatin and PCL/ gelatin/HAp microfibers were knitted together by crochet (Figure 5A, top), creating a uniform structure with diameters of $423.20 \pm 99.94 \mu \mathrm{m}$ for tendon (PCL/gelatin microfibers only), $410.45 \pm 56.06 \mu \mathrm{m}$ for the interface (PCL/gelatin plus PCL/gelatin/HAp microfibers) and $381.60 \pm 45.47 \mu \mathrm{m}$ for bone (PCL/gelatin/Hap microfibers only). Staining with AZ clearly showed a gradient of HAp (Figure 5A, bottom), being these observations confirmed by dye quantification of the different parts of the scaffold (Figure 5B). A significant increase was demonstrated by AZ quantification when comparing the different parts, from tendon to bone (tendon versus interface, $p<0.002$; interface versus bone, $p<0.00001$; and tendon versus bone, $p<0.00001)$. Additionally, micro-CT scanning was used to confirm the HAp content along the length of the fibrous scaffold (Figure 5C). Scan images allowed a clear visualization of a continuous and a gradual increase in HAp as observed in Figure $5 \mathrm{C}-\mathrm{i}$,ii. This increasing gradient was additionally confirmed by quantification of HAp content by micro-CT image analysis, which demonstrated a steady increase of mineral content along the scaffold, as observed in Figure 5D.

\subsection{Collagenous Matrix Production and Deposition}

Given the importance of collagen matrix deposition in tendon-to-bone interface, the production of collagen and noncollagenous proteins was assessed in gradient scaffolds. The collagen was examined across different sections of the scaffold (Figure 6B-i,ii) and semiquantification was performed for different sections the fibrous scaffolds, the tendon (PCL/ gelatin fibers only), interface (PCL/gelatin and PCL/gelatin/HAp fibers) and bony parts (PCL/gelatin/HAp only). Interestingly, quantitative data demonstrated higher collagen accumulation in the bony-part of the scaffold (****, $p<0.0001$, Figure 6C), even though the tendon extremity (only PCL/gelatin fibers) presented significantly higher collagen deposition in comparison with the interface $(p<0.014)$. Remarkably, when assessing noncollagenous proteins, the opposite was observed, where the highest accumulation occurred in the tendinous sections of the scaffolds ( $* * * *, p<0.0001$, Figure 6C).
A

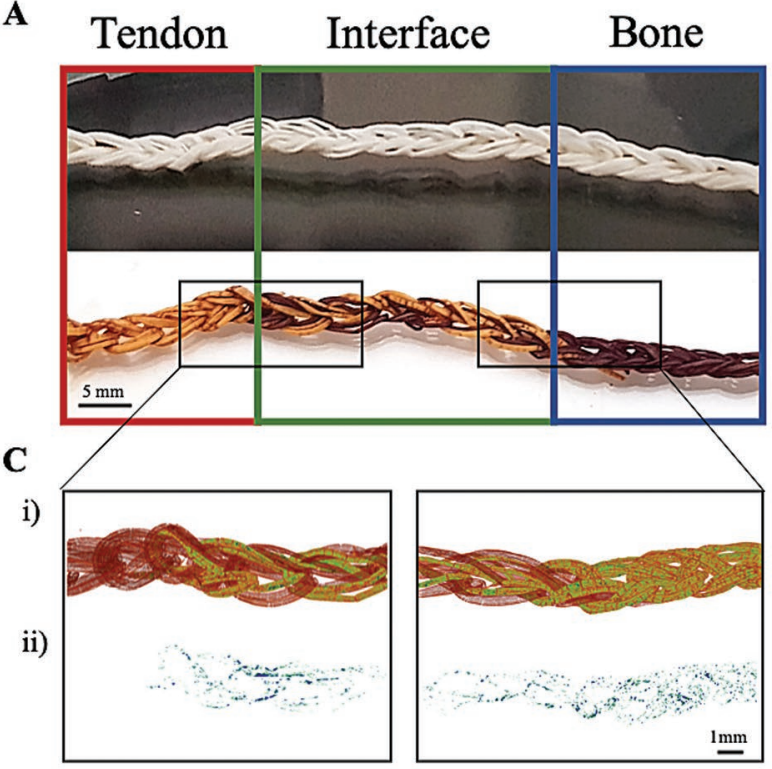

B

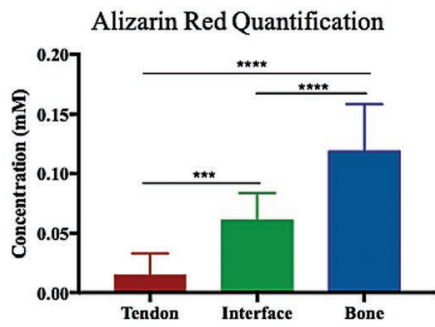

D

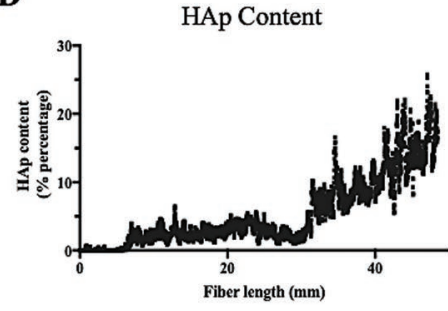

Figure 5. Morphology and composition of produced scaffolds with HAp gradient. A) 3D scaffolds were produced by crochet using PCL/gelatin and $\mathrm{PCL} /$ gelatin/HAp microfibers to mimic tendon, interface and bone. Scale bar, $5 \mathrm{~mm}$. B) Alizarin red staining was used to dye the scaffold showing a gradient in HAp content. (ii) Alizarin red quantification was performed using different sections of the scaffold through the cetylpyridinium method. Statistically significant differences are shown as $* * *, p<0.002$, $* * * *, p<0.00001, n=3$. C) Micro-CT scans of (i) different sections of the scaffold and (ii) HAp particles content. Scale bar, $1 \mathrm{~mm}$. D) Graphical demonstration of HAp content increase along the scaffold. 
$\mathbf{A}$

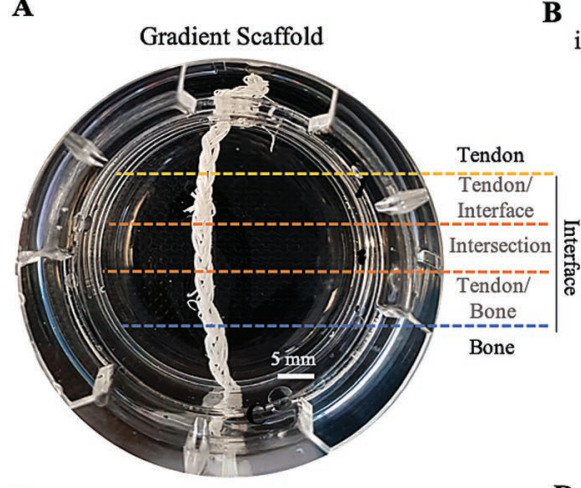

B i)

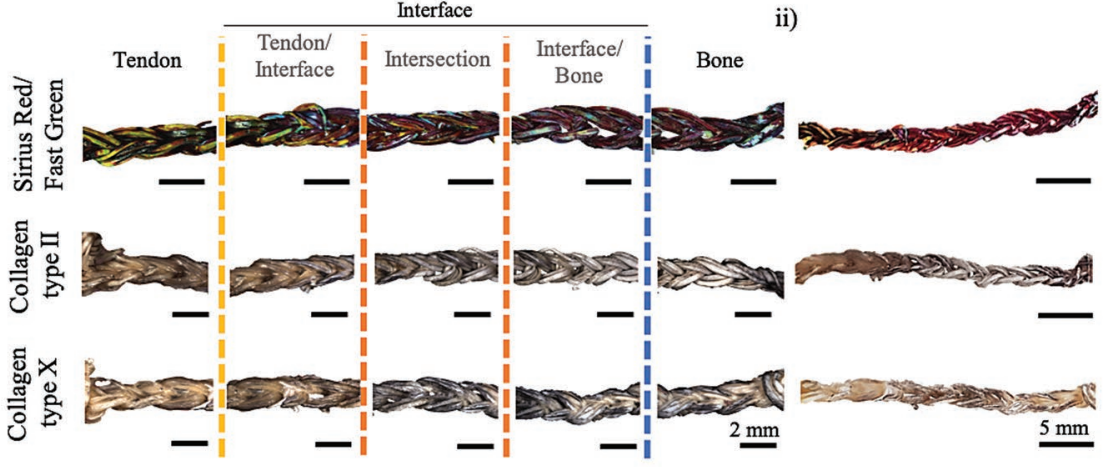

C

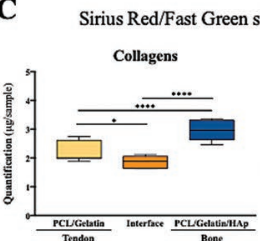

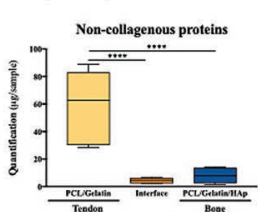

D
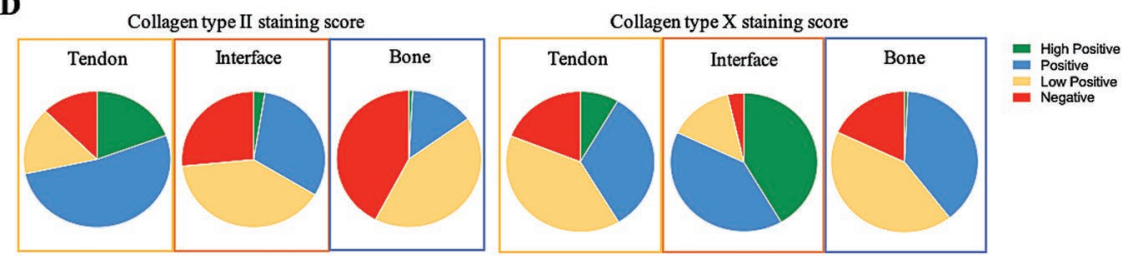

Figure 6. Histochemical staining of 3D gradient scaffolds. A) Gradient scaffolds were produced and seeded with hASCs for 14 days of culture in basal conditions. Scale bar, $5 \mathrm{~mm}$. B) Sirius red/fast green and immunostaining with DAB exposure (i-ii) were used to detect a gradient in collagens and noncollagenous proteins deposition and collagen types II and X along the fibrous scaffolds, respectively. Scale bar, $2 \mathrm{~mm}$ (i) and $5 \mathrm{~mm}$ (ii). C) Quantification of collagen (left) and non-collagenous proteins (right) was performed using different sections of the scaffold through the extraction of sirius red and fast green stain. Statistically significant differences are shown as $*, p<0.014$, *****, $p<0.00001 ; n=3$. D) DAB staining for collagen types II $(n=3)$ and $\mathrm{X}(n=3)$ was scored in several parts of scaffolds.

Additionally, the presence of collagens type II and X was also studied (Figure 6B-i,ii) due to their contribution to the nonmineralized and mineralized fibrocartilage regions of tendon-to-bone interface. Strikingly, it was observed a positive scoring of collagen type II in both tendon and interface sections of the scaffolds, while a low positive was obtained for the bonypart (Figure 6D). In contrast, highly positive staining score of collagen type $\mathrm{X}$ was found in the interface, while tendon and bone scored low positive and positive, respectively (Figure 6D).

\section{Discussion}

Macro- and microstructural features of developed biomaterials are known to affect cell survival and growth, as well as to play a key role in modulating cell phenotype and function. ${ }^{[17]}$ In this regard, new biomaterials for tendon-to-bone interface should mimic structural and compositional properties of this soft-hard tissue transition, comprising both nonmineralized and mineralized ECM components. In this work, two different polymeric solutions were used to mimic tendon (PCL/gelatin) and bone tissues (PCL/gelatin /HAp). Biodegradable synthetic polymers such as poly(lactic-co-glycolic acid) (PLGA), ${ }^{[18]}$ poly- $\varepsilon$-caprolactone (PCL), ${ }^{[12]}$ poly(lactic acid) (PLLA), ${ }^{[19]}$ have demonstrated suitability to wet-spinning processing. Among these, PCL has been widely used due to its compatibility, mechanical strength, low cost, and solubility in most solvents. However, its weak hydrophilicity affects cell adhesion and proliferation. Wet-spinning technique has been also widely used for processing natural origin polymers. The combination of PCL with natural polymers derived from ECM proteins, such as gelatin, has increased the biocompatibility of the fabricated fibers. ${ }^{[20]}$ Gelatin, which is derived from the controlled hydrolysis of collagen, is widely used in tissue engineering due to adequate low thermal stability, ${ }^{[10]}$ better solubility, and reduced antigenicity, in comparison to native collagen, as well as the presence of cell-binding motifs, like arginine-glycine-aspartic acid (RGD) sequences ${ }^{[21]}$ and MMP-sensitive degradation sites. ${ }^{[22]}$ Moreover, the addition of gelatin has been shown to result in more homogeneous fibers by electrospinning in comparison with pure PCL scaffolds. ${ }^{[23]}$ The formulations proposed herein have been previously used in the fabrication of electrospun scaffolds ${ }^{[23]}$ but these combinations have been relatively unexplored using wet-spinning as the fiber production method. Since natural polymers usually dissolve in aqueous environments, being gradually released, a coagulation bath with a crosslinking agent was included in the wet-spinning set-up to assure that gelatin was incorporated into the collected fiber. Glutaraldehyde is the most commonly used cross-linking agent, which has the ability to cross-link the primary amine groups of collagenous tissues. ${ }^{[24]}$ Even though percentages above $8 \%$ of glutaraldehyde have been reported to be cytotoxic, ${ }^{[25]}$ lowering the concentration has been reported to be biocompatible, as already described for anionic collagenous membranes. ${ }^{[26]}$ In this work, a solution of $1.25 \%$ (v/v) glutaraldehyde/water was used to produce the polymeric wet-spun microfibers. After injecting the polymeric solutions into the coagulation bath, composite microfibers could be collected in a continuous and large-scale manner. In addition, cell viability up to 14 days of culture suggests that the proposed system was harmless to seeded hASCs.

Mechanically, tendons and ligaments are demanding tissues exhibiting different mechanical properties depending on body location. ${ }^{[27]}$ At the interface, semitendinosus and Achilles tendons have higher young's modulus in the ranges of 540-1081 
and $819 \pm 208 \mathrm{MPa},{ }^{[28]}$ respectively. Typically, electrospun-based scaffolds proposed for tendon or ligament tissue engineering fail in meeting the native tissue's mechanical requirements, presenting tensile properties in lower ranges, normally with Young's modulus up to $30 \mathrm{MPa} \cdot{ }^{[29,30]}$ In this regard, produced wet-spun PCL/gelatin microfibers extruded at $1 \mathrm{~mL} \mathrm{~h}^{-1}$ have demonstrated to have an adequate mechanical performance, meeting the tendon mechanical requirements in a biomimetic manner.

Furthermore, to mimic as close as possible the composition of bone, micro-to-nanosized hydroxyapatite particles were incorporated in the polymeric solution. Hydroxyapatite is a well-established material for bone regeneration due to its biocompatibility, chemical resemblance with the inorganic part of bone and osteoconductive properties. Several studies have demonstrated that mesenchymal stem cells of both rat and human origins were sensitive to HAp concentrations as low as $1 \% \mathrm{w} / \mathrm{w}$ of solid scaffold weight and, increasing this concentration would result in different cells responses. ${ }^{[31]}$ Therefore, in this study, hydroxyapatite was used at percentages that trigger, in a controllable manner, an osteogenic-like cellular behavior without the need for osteogenic medium supplementation.

Additionally, topography has been shown to strongly affect cells response resulting in cells commitment to different lineages, which can be induced directly by the surface topography or from an altered ECM layer deposition on the surface of the biomaterial. ${ }^{[29,32,33]}$ In wet-spinning, fiber topography, as well as diameter can be tuned by changing the flow rate during injection. Therefore, three different extrusion flow rates were tested to study the topography of produced fibers. Interestingly, $1 \mathrm{~mL} \mathrm{~h}^{-1}$ extrusion flow rate seemed to induce a better alignment on PCL/gelatin microfibers, which is an important characteristic for the tendon counterpart of the junction, as already described in the literature. ${ }^{[29,32]}$ Several studies have shown that individual fiber anisotropy guides cell alignment potentially leading to a better control over pro-regenerative events, while 3D fibrous scaffolds can match the mechanical properties required at the tissue level. ${ }^{[32,34]}$ Herein, the aligned arrangement of the PCL/gelatin wet-spun composite microfibers induced a high cytoskeleton alignment and nuclei elongation, resembling native tendon anisotropic organization. This was observed after seeding hASCs, which were able to align following the direction of the surface topography of PCL/gelatin microfibers through contact guidance mechanisms, even after prolonged times in culture.

In contrast, PCL/gelatin/HAp microfibers produced at the same extrusion rate presented HAp particles distributed along the surface of the fiber, sticking out and creating lumps and beads. This morphology resulted from the presence of larger sized particles as observed by the micro-CT analysis. Moreover, changing the flow rate also affected the porosity of PCL/gelatin/ HAp fibers. Extrusion of fibers at $1 \mathrm{~mL} \mathrm{~h}^{-1}$, resulted in higher pore volumes and pore amount. This may be correlated with the amount of polymeric solution extruded, meaning that a high flow rate leads to higher amounts of polymeric solution to be extruded to the crosslinking bath, which resulted in the accumulation of HAp particles in the surface of larger fibers. The same was not observed for lower extrusion rates, where the amount and time of polymeric solution in contact with the bath are decreased, leading to thinner fibers incorporating small sized particles.

In a study by Fibers topography and flow rate can potentially be correlated to the "Kelvin-Helmholtz instability" Equation (1), as demonstrated elsewhere: ${ }^{[35]}$

$(U 1-U 2)^{\wedge} 2>2 \sqrt{ } g \sigma\left(\rho 1-\rho 2(\rho 1+\rho 2)\left(\frac{1}{\rho 1 \rho 2}\right)\right)$

where, $\rho 1, \rho 2, U 1$, and $U 2$ are the densities and velocities of liquids 1 and 2, $g$ is the acceleration due to gravity, and $\sigma$ is the interfacial tension between the two fluids. In this study conditions, $U 2$ tends to 0 as it corresponds to the coagulation bath.

Likewise, fibers diameter can also be correlated with the flow rate as described by Han et al., ${ }^{[36]}$ who demonstrated that the volumetric flow rate is directly proportional to the filament cross-sectional area Equation (2)

$A(x)=\frac{Q}{V(x)}$

where $A(x)$ is the filament cross-sectional area, $V(x)$ is the axial velocity, and $Q$ is the volumetric flow rate. Similarly, this is also observed in electrospinning technique, where the flow rate influences both the diameter and morphology of fibers, meaning that high flow rate results in longer drying time due to greater volume of polymeric solution drawn from the needle. ${ }^{[37]}$

Incorporation of HAp decreased the Young's modulus of PCL/gelatin/HAp microfibers in comparison with PCL/gelatin wet spun fibers, according to what has been previously reported. ${ }^{[38]}$ Moreover, the presence of HAp led to a higher matrix mineralization as soon as 7 days, suggesting an induction of osteogenic differentiation of hASCs. Results from the literature show that the micropattern of hydroxyapatite in materials seems to induce a higher promotion in early cells adhesions and subsequent osteogenic differentiation. ${ }^{[39]}$

The fabrication of a suitable interface engineered approach is based on the design of stratified or multigraded biomimetic scaffolds able to replicate the complex and hierarchical organization, structure, and mechanical properties present in tendon-to-bone junction. ${ }^{[40]}$ Great attention has been given to the potential use of textile technologies for the biofabrication of tissue engineered constructs. Different textile techniques, among knitting, weaving, braiding, can be used to create fibrous structures with the demanded properties of different tissues, using a rational assembly of fibers into bigger textile constructs while conserving the main features of the single unit. ${ }^{[41]}$ In tendon-to-bone interface, changes in structure, composition, and mineralization are gradual and continuous and aid an efficient transfer of load between the two dissimilar tissues. ${ }^{[42]}$ Thus, taking advantage of textile techniques, the produced polymeric fibers (PCL/gelatin and PCL/gelatin/HAp, $1 \mathrm{~mL} \mathrm{~h}^{-1}$ ) were knitted together by crochet to create a gradient in composition along the scaffold. This technique differs from knitting because the stitches are built on top of each other and the active loop is the only place from which the scaffold is prone to unraveling, making the construct more stable. To mimic the tendon structure, PCL/gelatin fibers were crochet together and afterwards, 
to create the interface, PCL/gelatin/HAp microfibers were gradually incorporated until only this material was left. Alizarin red staining and HAp profile from micro-CT allowed to confirm the presence of a mineral gradient in HAp content. Overall, these results show that engineered microfibers could be handled manually and were able to withstand the textile assembling to produce 3D gradient scaffolds.

Tendon-to-bone interface is also characterized by the presence of four different zones (tendon, nonmineralized, and mineralized fibrocartilage and bone), with collagen as major component. ${ }^{[43]}$ These transitions were also observed in produced fibrous scaffolds. Strikingly, it was possible to distinguish four different zones: tendon (first sections), interface which was composed by the tendon/intersection and intersection/bone, and bone (last section). Taking advantage of the topological and compositional differences across the gradient scaffolds, it was possible to induce the deposition of different collagens in the distinctive parts of the construct. Even though collagens were quantified in all parts, noncollagenous proteins were highly present in the tendon section. In tendon, collagen fibers are interspersed by a variety of noncollagenous matrix components, namely proteoglycans, glycoproteins, and glycoconjugates, playing an important role within the tissue. ${ }^{[4]}$ The presence of collagen type II and collagen type X was observed in the interfaced section, as demonstrated by the score obtained along the scaffolds. This is also observed in the native tissue, where nonmineralized fibrocartilage is characterized by a collagenous matrix composed of collagen types II and III, with small amounts of types I, IX, and X collagen. ${ }^{[45]}$ The mineralized fibrocartilage also contains collagen type II, with significant higher amounts of type $X$ collagen. ${ }^{[45]}$

A similar work was developed by Sun et al. ${ }^{[46]}$ in which coelectrospun dual scaffolds, poly(lactic-co-glycolic acid)/collagen I-polycaprolactone/nanohydroxyapatite (PLGA/Col-PCL/nHA), were used to bridge massive rotator cuff tears in rabbit models. ${ }^{[46]}$ However, the authors did not truly generate a gradient of mineral and collagen composition, which is addressed by the gradient developed in the present study. On the other hand, Li et al. ${ }^{[4]}$ developed gradients of mineral content in PCL and PLGA nanofibers using concentrated simulated body fluid. However, only randomly aligned nonwoven mats coated with calcium phosphate were used to mimic the tendon-to-bone insertion site, not truly mimicking the topological features of the native multitissue transition. Moreover, gradient scaffolds were not able to uniformly support preosteoblast cells adhesion. ${ }^{[47]}$ Comparably, Nowlin et al. ${ }^{[48]}$ produced randomto-aligned nanofiber scaffolds to mimic the microtopology of tendon-to-bone interface. Even though a transitional region was obtained, authors seeded terminally cells (fibroblasts and osteosarcoma cells) on the different regions and did not demonstrate the ability of cells to differentiate toward specific lineages due to the influence of fibers topology and composition.

Overall, produced scaffolds demonstrated the potential use in tendon-to-bone tissue regeneration by replicating the transition between tissues and collagen matrix deposition observed in the native interface.

In summary, through the addition of HAp into the initial polymeric solutions, structural and compositional properties could be tuned. The developed microfibers can potentially guide a single cell source toward different behaviors. Cellular morphometric characteristics demonstrated that the topography of $\mathrm{PCL} /$ gelatin wet-spun composite microfibers could induce a preferential alignment of seeded hASCs, resembling the anisotropic cellular organization found in native tendon niches. Matrix mineralization on PCL/gelatin/HAp wet-spun composite microfibers suggested that seeded hASCs were able to produce an osteogenic-like ECM, without the external addition of any osteoinductive factors. Altogether, these results demonstrate the feasibility of using wet-spinning method to generate continuous aligned and textured composite microfibers, which can be further assembled into more complex 3D gradient structures through the application of textile techniques to closely resemble tendon-to-bone microstructure.

\section{Conclusion}

Tendon-to-bone interface is a complex hierarchical tissue, exhibiting unique structural and compositional characteristics. The development of advanced tissue engineered approaches should focus the use of straightforward techniques toward mimicking the native features of the different tissues forming the junction. In this study, a wet-spinning technique was used to produce two different wet spun microfibers with compositional and structural differences in order to mimic tendon anisotropic organization and mineral composition of bone. Biological studies revealed that the fibers were not only able to support cell proliferation but also to favor cellular anisotropic alignment or to induce an osteogenic-like phenotype on human adipose derived stem cells in the presence of HAp. In fact, the continuous wetspinning technique in combination with textile technologies enabled the generation of a fibrous scaffold with a gradient in polymer composition, resulting in a mineral gradient of HAp content. Altogether, results clearly demonstrated the feasibility of using simple fiber processing techniques, such as wet-spinning, to tailor cells response while having a precise control over fibers topography and composition. Moreover, the combination with advanced textile techniques allowed the development of 3D fibrous scaffolds envisioning tendon-to-bone regeneration.

\section{Experimental Section}

Materials: Poly- $\varepsilon$-caprolactone (PCL, average $M_{n}=80$ 000), gelatin (type A, from porcine skin), phosphate buffered saline (PBS), 4,6-diamidino-2-phenyindole dilactate (DAPI, $5 \mu \mathrm{g} \mu \mathrm{L}^{-1}$ ), bovine serum albumin (BSA), and Cellcrown 24-well plate inserts were purchased from Sigma-Aldrich (Germany). Formic acid $98 \%$ was purchased from Laborspirit (Spain). Glacial acetic acid and 25\% (v/v) glutaraldehyde were obtained from VWR (Belgium). Antibiotic/antimycotic solution $(\mathrm{A} / \mathrm{A})$, fetal bovine serum (FBS), and minimum essential medium alpha $(\alpha$-MEM) were purchased from Alfagene (United Kingdom). PhalloidiniFluor conjugate was purchased from ATT Bioquest (USA). Basal medium was prepared using $\alpha$-MEM supplemented with $10 \%(\mathrm{v} / \mathrm{v})$ FBS and $1 \%(\mathrm{v} / \mathrm{v}) \mathrm{A} / \mathrm{A}$.

Hydroxyapatite Nano-to-Microparticles Synthesis (HAp): A precipitation reaction of calcium hydroxide (Riedel-de Haën, Germany) and orthophosphoric acid $85 \%$ (Panreac, Spain) solutions was performed in an aqueous system at room temperature in order to obtain HAp. The mixing was performed in a basic environment $(\mathrm{pH} 11)$, adjusted with concentrated ammonium hydroxide (1 M, Riedel-de Haën, Germany) 
at a continuous flow rate ranging from 8 to $15 \mathrm{~mL} \mathrm{~min}$. . The formed ceramic powder was sieved to obtain particles smaller than $63 \mu \mathrm{m}$ X-ray.

Fabrication of Continuous PCL/Gelatin and PCL/Gelatin/HAp Fibers by Wet Spinning: A PCL solution (22\% w/v, initial concentration) was prepared by dissolving PCL in formic acid/acetic acid (3:1 v/v) and stirring at $150 \mathrm{rpm}$ for $4 \mathrm{~h}$. Gelatin $(9 \% \mathrm{w} / \mathrm{v}$, initial concentration) was dissolved in a solution of $80 \%$ acetic acid/water ( $\mathrm{v} / \mathrm{v})$ and stirred at $150 \mathrm{rpm}$ for $4 \mathrm{~h}$. The final solution of PCL/gelatin was prepared by mixing $P C L$ and gelatin solutions at a ratio of $70: 30$, respectively. In the case of PCL/gelatin/HAp blend, HAp nano-to-microparticles were first completely dispersed in PCL solution and, afterwards, gelatin $(9 \% \mathrm{w} / \mathrm{v})$ solution was added to the mixture. HAp content represents $7.7 \% \mathrm{w} / \mathrm{v}$ of the final solution. Both solutions were prepared at room temperature and used within the day of preparation. Fibers were fabricated using the wet-spinning method. A customized device was used to produce textured wet-spun microfibers. A coagulation bath containing $1.25 \%$ $(\mathrm{v} / \mathrm{v})$ glutaraldehyde/water solution was used as crosslinker. The prepared solutions were injected using a $10 \mathrm{~mL}$ syringe with a $21 \mathrm{G}$ needle and extruded to the coagulation bath at a constant flow rate. Three different flow rates were tested: $1,0.5$, and $0.25 \mathrm{~mL} \mathrm{~h}$. The vertical distance from the needle to the surface of the coagulation bath (Figure 1A) was set at $6 \mathrm{~cm}$. A collector was set after the coagulation bath at a horizontal distance of $8 \mathrm{~cm}$ from the needle and the fibers were collected at a constant speed (two rotations per minute) (Figure 1A). All fibers were left inside the hood overnight to eliminate possible solvent and/or glutaraldehyde residues from the production process.

Morphological Characterization: Fibers obtained from different formulations and flow rates were observed and photographed under an optical microscope. Fiber diameter was determined by measuring three different regions in the images. Images were obtained from six independent samples per condition. The morphology of the different fibers was analyzed by scanning electron microscopy (SEM, MP1000001280128, JSM-6010 LV, JEOL). All samples were previously coated with $2 \mathrm{~nm}$ of platinum (Cressigton). The degree of surface alignment was determined through directionality analysis applying the Fourier components method using the FIJI software (version 2.0.0). For this purpose, scanning electron images were converted to 32-bit images and cropped into three different images. The radial intensities were calculated, and the orientation map was also obtained.

In order to evaluate its microstructure, single microfibers of $\mathrm{PCL}$ / gelatin/HAp were analyzed by $\mathrm{X}$-rays micro-computed tomography (micro-CT) using a high-resolution X-ray microtomography system was used: Skyscan 1 scanner (Skyscan 1272; Bruker, Billerica, MA, USA). The acquisition of X-ray images was performed with a pixel size of $10 \mu \mathrm{m}$, a rotation step of $0.4^{\circ}$ over $360^{\circ}$, and a smoothing averaging of every three images. The X-ray source was fixed at $50 \mathrm{keV}$ and $200 \mu \mathrm{A}$, of voltage and current, respectively. Following the acquisition, gray-scale images were reconstructed using the NRecon software (version 1.7.1.0, Bruker, Billerica, MA, USA). Then, the samples were vertically aligned for the longitudinal analysis of the fibers using the DataViewer software (version 1.5.3.6, Bruker, Billerica, MA, USA). Qualitative visualization of the 3D morphology and the different phases of the polymer and hydroxyapatite were performed using CT-Vox software (version 3.3.0, Bruker, Billerica, MA, USA). Finally, the quantitative analysis was performed after converting the regions of interest into binary images, by a dynamic threshold (30-255polymeric phase; $80-255$ ceramic phase). So, the binary images were used for morphometric examination (CT Analyzer v1.12.0.0, SkyScan, Kontich, Belgium) of porosity amount, pore volume, and interconnectivity.

Chemical Characterization: Fourier-transform infrared spectroscopy (FTIR, IRPrestige 21, Shimadzu) was used for chemical analysis of the developed fibers. Briefly, all samples were mixed with potassium bromide $(\mathrm{KBr})$ and pressed into transparent pellets prior to FTIR analysis. Infrared spectra of $\mathrm{PCL}$, gelatin, $\mathrm{Hap}$, and $\mathrm{PCL} /$ gelatin, and $\mathrm{PCL} /$ gelatin/HAp fibers were recorded by averaging 32 individual scans, at a resolution of $4 \mathrm{~cm}^{-1}$, over a wavenumber range between 4000 and $400 \mathrm{~cm}^{-1}$ at room temperature.

Mechanical Characterization: Mechanical properties of produced wet spun fibers were assessed using the universal mechanical testing machine (5543K2942, 5543, Instron) equipped with a $1 \mathrm{kN}$ load cell. Images from optical microscope (DM750, Leica, Schweiz, Germany) were acquired and used for cross-sectional area calculations. Each specimen was measured along the length at three different locations using the Image) software (version 1.52d). Samples were cut into testing specimens with $1.5 \mathrm{~cm}$ and fixed in paper frames with a window of $1.2 \times 1.2 \mathrm{~cm}$. After mounting the frames onto the tester grips, the lateral sides were cut and the crosshead speed was set at a constant rate of $1 \mathrm{~mm} \mathrm{~min}{ }^{-1}$. At least six samples per condition were tested. The elastic modulus was calculated from the tangent slope of the linear section of the stress-strain curve.

Cell Seeding: Human-adipose derived cells (hASCs) were isolated by enzymatic digestion of lipoaspirate samples obtained under previously established protocols with Hospital da Prelada (Porto, Portugal), as described elsewhere. ${ }^{[49]}$ Human lipoaspirate samples were collected under informed consent following protocols approved by the ethical committee of the hospital and according to the Declaration of Helsinki. Human lipoaspirate samples $(n=3)$ of healthy female patients with ages in the range of 60-70 were used. Isolated cells were cultured and expanded in basal medium.

Prior to seeding, both wet-spun fibers and fibrous scaffolds were immersed in $70 \%$ ethanol for $15 \mathrm{~min}$, followed by a washing step with PBS for 15 min to remove the remaining ethanol residues.

Single fibers were then fixed inside 24-well plate inserts and left overnight in basal medium. Afterward, after removing the medium, hASCs were seeded on the fibers at a density of $2 \times 10^{4}$ cells using $400 \mu \mathrm{L}$ per well in basal medium. After $1 \mathrm{~h}$ of incubation in a humidified environment $\left(37^{\circ} \mathrm{C}, 5 \% \mathrm{CO}_{2}\right)$, culture medium was added up to $1 \mathrm{~mL}$.

Scaffolds were fixed with six-well plate inserts and left overnight with basal medium. Cells were seeded on the scaffolds at a density of $1 \times 10^{5}$ cells using $1 \mathrm{~mL}$ per well in basal medium. After $1 \mathrm{~h}$ of incubation $\left(37^{\circ} \mathrm{C}, 5 \% \mathrm{CO}_{2}\right)$, basal culture medium was added up to $4 \mathrm{~mL}$. Cells were used at passage $2-3$ and culture medium was changed twice a week over 14 days of culture.

Determination of Metabolic Activity by Alamar Blue Assay: After 1, 7, and 14 days in culture, the metabolic activity of hASCs was evaluated by Alamar blue assay, according to manufacturer's instructions (AbD SeroTec, Bio-Rad). Briefly, cell-seeded fibers were transferred to clean wells and washed twice with PBS. Then, $10 \%(\mathrm{v} / \mathrm{v})$ Alamar blue in basal medium was added and cells were incubated overnight under standard culture conditions. The supernatant was collected, and the fluorescence measured using a microplate reader (Synergy HT, Bio-Tek Instruments) at an excitation wavelength of $530 \mathrm{~nm}$ and an emission wavelength of $590 \mathrm{~nm}$. Quadruplicates were used for each fiber.

Alizarin Red (AZ) Staining and Quantification: Matrix mineralization was analyzed by alizarin red staining after 7 and 14 days of culture. Briefly, fibers were fixed with $10 \%(\mathrm{v} / \mathrm{v})$ neutral buffered formalin for $20 \mathrm{~min}$ at room temperature (RT). Afterwards, samples were washed with PBS and deionized water. Subsequently, a solution of $2 \%(\mathrm{w} / \mathrm{v})$ AZ (Merck) was added for $10 \mathrm{~min}$ and the excess of dye removed with deionized water. Staining was visualized and images were acquired using the inverted microscope Vert A.1 Axio with Axiocam 503 color (Zeiss, Germany) and Zen 2.3 lite software (Zeiss, Germany). Quantitative data were obtained using the cetylpyridinium chloride (CPC) method. The dye was removed using a solution of $10 \%(\mathrm{w} / \mathrm{v}) \mathrm{CPC}$ in $10 \times 10^{-3} \mathrm{M}$ sodium phosphate (Sigma-Aldrich) for $1 \mathrm{~h}$, under gentle shaking, at RT and the absorbance measured at $562 \mathrm{~nm}$ in a microplate reader (Synergy HT, Bio-Tek Instruments). Fibers without cells were used as controls and the dye quantification used as background control for fibers with cells. Triplicates were used for each sample and conditions.

Morphological Evaluation of Seeded hASCS by SEM: The organization and morphology of hASCs cultured on developed microfibers were analyzed by SEM. Briefly, after 7 and 14 days, cell-seeded fibers were washed three times with PBS, fixed with $2.5 \%(\mathrm{v} / \mathrm{v})$ glutaraldehyde in PBS solution and stored at $4{ }^{\circ} \mathrm{C}$ until preparation. After this, all samples were dehydrated in a series of increasing concentrations of ethanol in water (10\% to $100 \%, 30$ min of incubation in each solution). After dehydration, all samples were left to air-dry and mounted on aluminum 
stubs. Prior to SEM analysis, a coating with platinum was performed and the samples were observed at an accelerating voltage of $5 \mathrm{kV}$.

Immunofluorescence and F-Actin Staining: After 7 and 14 days of culture, fibers seeded with hASCs were washed with PBS and fixed with $10 \%(\mathrm{v} / \mathrm{v})$ neutral buffered formalin for $20 \mathrm{~min}$ at RT. To assess the deposition of collagen type III, fibers were first washed with PBS and permeabilized with $0.25 \%$ Triton X-100/PBS solution for 5 min. To block nonspecific binding, a solution of $1 \%$ bovine serum albumin $(\mathrm{w} / \mathrm{v}, \mathrm{BSA})$ in PBS was added and left for $30 \mathrm{~min}$ at RT. Samples were afterwards incubated overnight with rabbit anti-human collagen type III (COLIII, 1:100, Abcam, ab7778). After incubation with the primary antibody, mouse anti-rabbit AlexaFluor 488 was used as secondary antibody. All antibodies were diluted in $1 \%(\mathrm{w} / \mathrm{v}) \mathrm{BSA}$. Cell nuclei were counterstained with 4,6-diamidino-2-phenyindole dilactate (DAPI, Invitrogen) in a dilution of 1:1000. All samples were visualized, and images acquired using Z-stack method by fluorescence microscopy (Axio Imager Z1m, Zeiss, Deutschland, Germany).

Actin filaments of cell cytoskeleton were stained with phalloidin. Briefly, samples were washed three times with PBS after formalin fixation and incubated with phalloidin solution for $90 \mathrm{~min}$ at room temperature. Then, samples were washed with PBS and analyzed by confocal laser scanning microscopy (Leica TCS SP8, Microsystems, Wetzlar, Germany).

Organization of Actin Filaments and Nuclei Elongation: The degree of actin filament alignment was determined through directionality analysis using the Fourier components method using the FIJI software (version 2.0.0). For this purpose, confocal images were converted to 32-bit images and cropped into three different images. The radial intensities were calculated, and the orientation map was also obtained. Nuclei aspect ratio was determined measuring at least 40 nuclei in different labeled images of each fiber, using Image) software (version 1.52d). The length was divided by the width to obtain the correspondent aspect ratio.

Assembly of a 3D Gradient Scaffold Using Textile Techniques: To fabricate a scaffold with a gradient of HAp content, knitting (crochet) was used as the main textile technique. Briefly, fibers from each formulation produced at $1 \mathrm{~mL} \mathrm{~h}{ }^{-1}$ were used. First, four PCL/gelatin fibers were knot together and, with a hook needle, over hook and draw through to form a new loop without tightening the previous one, forming a chain stitch. In the middle region of the 3D scaffold, two $\mathrm{PCL} /$ gelatin fibers were removed, and two fibers of $\mathrm{PCL} /$ gelatin/HAp were interlocked with the remaining ones to produce the interface. Finally, $\mathrm{PCL} /$ gelatin fibers were completely removed to obtain a third part of the scaffold comprising only $\mathrm{PCL} /$ gelatin/HAp fibers. The mineral content along the gradient scaffold was determined by alizarin red staining and quantification methods, as described above.

Immunohistochemical Staining of Gradient Scaffolds: After 14 days of culture scaffolds seeded with hASCs were washed with PBS and fixed with $10 \%(\mathrm{v} / \mathrm{v})$ neutral buffered formalin for $20 \mathrm{~min}$ at RT. For the detection of collagen type II and $\mathrm{X}$ protein, scaffolds were washed with PBS and quenched of peroxidase activity with $3 \%$ oxygen peroxide $\left(\mathrm{H}_{2} \mathrm{O}_{2}\right)$ solution in water. Afterwards, samples were blocked with $2.5 \%$ normal horse serum for $30 \mathrm{~min}$ at RT (VECTASTAIN Elite ABC HRP Kit, Vector Lab, UK) and incubated either with mouse anticollagen type II (COLIII, 1:200, Merck, MAB1330) or rabbit anticollagen type X (COLX, 1:50, Abcam, ab58632) for $2 \mathrm{~h}$ at RT. The secondary antibody was then applied for $30 \mathrm{~min}$. Samples color was developed using the Vectastain $A B C$ reagent for $30 \mathrm{~min}$, followed by an exposure to 3,3-diaminobenzidine substrate (DAB) for 3 min (DAB Peroxidase (HRP) Substrate Kit (with Nickel), Vector Lab, UK). Stained samples were visualized, and images acquired under a stereomicroscope (Stemi 2000-C, Zeiss, Deutschland, Germany) with AxioCam ICCl (Zeiss, Germany). Quantitative data were obtained using the IHC profiler plugin of Image) software (version $1.52 \mathrm{~d}$ ). Scaffolds made of only PCL/gelatin and PCL/gelatin/HAp were used as controls. Duplicates were used and data acquired from three different parts of each image/section.

Semiquantitative Analysis of Collagen and Noncollagenous Proteins: To detect collagen and noncollagenous proteins across the scaffolds, Sirius red/fast green staining kit (Chondrex, Inc., Washington, USA) was used in fixed samples according manufacturer's instructions. Briefly, after 14 days of culture, scaffolds fixed with $10 \%(\mathrm{v} / \mathrm{v})$ neutral buffered formalin for $20 \mathrm{~min}$ at room temperature (RT) and washed with PBS, were completely submersed in a dye solution for $1 \mathrm{~h}$ at RT. Afterwards, the excess dye was rinsed off with distilled water and images acquired using a stereomicroscope (Stemi 2000-C, Zeiss, Deutschland, Germany) with AxioCam ICCl (Zeiss, Germany). Semiquantification was performed trough the dye extraction with a buffer and the OD reading taken at 540 and $605 \mathrm{~nm}$ using a microplate reader (Synergy HT, Bio-Tek Instruments). Collagen and noncollagen protein content was calculated from the formulae provided by the supplier. Triplicates were used for each section. $\mathrm{PCL} /$ gelatin and PCL/gelatin/HAp scaffolds were used as controls.

Morphological Characterization of the Scaffold: Developed 3D fibrous scaffolds were analyzed by micro-computed X-ray tomography ( $\mu-C T)$. Several images from different parts of the fibrous scaffold were acquired by X-ray diffraction, and then used for analysis and 3D reconstruction. The HAp profile was traced and quantified. 3D projections of the specimens were performed. The 3D gradient structures were acquired with a SkyScan 1272 scanner (v1.1.3, Bruker, Boston, USA), with a pixel size of $11 \mu \mathrm{m}$. Approximately 1300 to 1700 projections were acquired over a rotation angle of $360^{\circ}$, with a rotation step of $0.20^{\circ}$. Data sets were reconstructed using a standardized cone-beam reconstructed software (NRecon v1.7.1.0, Bruker). Bitmap images were obtained as a final output. Representative dataset of the slices was segmented into binary images with a dynamic threshold of $\approx 100$ to 255 for the analysis of the ceramic phase, and from 16 to 255 for soft polymeric phase (grey scale values-optimized per sample and analysis). Then, the binary images were used for morphometric analysis (CT Analyzer, v1.17.0.0, Bruker) and to build the 3-D models (CTvox, v 3.3.0, Bruker). When needed, samples were oriented in DataViewer (v1.5.3.6, Bruker) before proceeding to CT Analyzer and CTvox.

Statistical Analysis: Results were obtained from three independent experiments with a minimum of three replicates for each condition. Results are presented as mean \pm standard deviation (SD). Statistical analyses were performed using GaphPad Prism 7.0 software. Two-way analysis of variance (ANOVA) with Sidak and Tukey tests and multiple comparisons with Tukey tests were performed. Differences between experimental groups were considered significant with a confidence interval of $95 \%$, whenever $p<0.05$.

\section{Supporting Information}

Supporting Information is available from the Wiley Online Library or from the author.

\section{Acknowledgements}

The authors thank to Hospital da Prelada (Porto, Portugal) for providing lipoaspirate tissue (Plastic Surgery Department) samples; Dr. Rui Domingues and Dr. Pedro Babo for the technical help with confocal microscopy and micro-CT analysis. The authors acknowledge the financial support from the European Union Framework Programme for Research and Innovation HORIZON2020, under the TEAMING Grant agreement No 739572 - The Discoveries CTR, the ERC Grant CoG MagTendon nr 772817, FCT-Fundação para a Ciência e a Tecnologia for the PhD grant of IC (PD/BD/128088/2016); and the Project NORTE-01-0145-FEDER-000021: "Accelerating tissue engineering and personalized medicine discoveries by the integration of key enabling nanotechnologies, marine-derived biomaterials and stem cells," supported by Norte Portugal Regional Operational Programme (NORTE 2020), under the PORTUGAL 2020 Partnership Agreement, through the European Regional Development Fund (ERDF).

\section{Conflict of Interest}

The authors declare no conflict of interest. 


\section{Keywords}

biotextiles, cell-laden microfibers, gradient biomaterials, tendon-to-bone interfaces, wet spinning

Received: February 14, 2019 Revised: May 20, 2019 Published online:

[1] K. A. Derwin, A. R. Baker, J. P. Iannotti, J. A. McCarron, Tissue Eng., Part B 2010, 16, 21.

[2] a) S. Thomopoulos, J. Biomech. Eng. 2003, 125, 106; b) S. Thomopoulos, G. Hattersley, V. Rosen, M. Mertens, L. Galats, G. R. Williams, L. J. Soslowsky, J. Orthop. Res. 2002, 20, 454.

[3] A. G. Schwartz, J. D. Pasteris, G. M. Genin, T. L. Daulton, S. Thomopoulos, PLoS One 2012, 7, 1.

[4] A. Tamayol, M. Akbari, N. Annabi, A. Paul, A. Khademhosseini, D. Juncker, Biotechnol. Adv. 2013, 31, 669.

[5] a) J. Burck, S. Heissler, U. Geckle, M. F. Ardakani, R. Schneider, A. S. Ulrich, M. Kazanci, Langmuir 2013, 29, 1562; b) L. Yang, C. F. Fitie, K. O. van der Werf, M. L. Bennink, P. J. Dijkstra, J. Feijen, Biomaterials 2008, 29, 955.

[6] D. R. Nisbet, J. S. Forsythe, W. Shen, D. I. Finkelstein, M. K. Horne, J. Biomater. Appl. 2009, 24, 7.

[7] E. Mathiowitz, M. D. Lavin, R. Hopkins, Ther. Delivery 2013, 4, 1075.

[8] J. M. Caves, V. A. Kumar, J. Wen, W. Cui, A. Martinez, R. Apkarian, J. E. Coats, K. Berland, E. L. Chaikof, J. Biomed. Mater. Res., Part B 2010, 93, 24.

[9] D. Puppi, A. M. Piras, F. Chiellini, E. Chiellini, A. Martins, I. B. Leonor, N. Neves, R. Reis, J. Tissue Eng. Regener. Med. 2011, 5, 253.

[10] D. Puppi, F. Chiellini, Polym. Int. 2017, 66, 1690.

[11] S. C. Neves, L. S. Moreira Teixeira, L. Moroni, R. L. Reis, C. A. Van Blitterswijk, N. M. Alves, M. Karperien, J. F. Mano, Biomaterials 2011, 32, 1068

[12] D. Puppi, C. Mota, M. Gazzarri, D. Dinucci, A. Gloria, M. Myrzabekova, L. Ambrosio, F. Chiellini, Biomed. Microdevices 2012, 14, 1115

[13] Y. Yang, J. Sun, X. Liu, Z. Guo, Y. He, D. Wei, M. Zhong, L. Guo, H. Fan, X. Zhang, Regener. Biomater. 2017, 4, 299.

[14] S. W. Volk, S. R. Shah, A. J. Cohen, Y. Wang, B. K. Brisson, L. K. Vogel, K. D. Hankenson, S. L. Adams, Calcif. Tissue Int. 2014, 94, 621.

[15] C. S. Chamberlain, E. M. Crowley, H. Kobayashi, K. W. Eliceiri, R. Vanderby, Microsc. Microanal. 2011, 17, 779.

[16] H. C. Blair, Q. C. Larrouture, Y. Li, H. Lin, D. Beer-Stoltz, L. Liu, R. S. Tuan, L. J. Robinson, P. H. Schlesinger, D. J. Nelson, Tissue Eng., Part B 2017, 23, 268.

[17] a) J. Leijten, J. Rouwkema, Y. S. Zhang, A. Nasajpour, M. R. Dokmeci, A. Khademhosseini, Small 2016, 12, 2130; b) W. Liu, J. Lipner, J. Xie, C. N. Manning, S. Thomopoulos, Y. Xia, ACS Appl. Mater. Interfaces 2014, 6, 2842.

[18] K. Wanawananon, S. E. Moulton, G. G. Wallace, S. Liawruangrath, Polym. Adv. Technol. 2016, 27, 1014.

[19] J. S. Fernandes, R. L. Reis, R. A. Pires, Mater. Sci. Eng., C 2017, 71, 252.

[20] J. Lee, G. Tae, Y. H. Kim, I. S. Park, S.-H. Kim, S. H. Kim, Biomaterials 2008, 29, 1872.

[21] Y. Liu, M. B. Chan-Park, Biomaterials 2010, 31, 1158.

[22] J. Vandooren, P. E. Van den Steen, G. Opdenakker, Crit. Rev. Biochem. Mol. Biol. 2013, 48, 222.

[23] M. Sattary, M. T. Khorasani, M. Rafienia, H. S. Rozve, Polym. Adv. Technol. 2018, 29, 451.

[24] L. H. H. O. Damink, P. J. Dijkstra, M. J. A. Van Luyn, P. B. Van Wachem, P. Nieuwenhuis, J. Feijen, J. Mater. Sci. 1995, 6, 450.
[25] P. R. Umashankar, P. V. Mohanan, T. V. Kumari, Toxicol. Int. 2012, 19, 51.

[26] G. Goisis, E. M. Junior, A. C. Marcantonio, R. C. C. Lia, D. C. J. Cancian, W. M. Carvalho, Biomaterials 1999, 20, 27.

[27] J. G. Barber, A. M. Handorf, T. J. Allee, W. J. Li, Tissue Eng., Part A 2013, 19, 1265.

[28] H.-J. Jung, M. B. Fisher, S. L. Y. Woo, BMC Sports Sci. Med. Rehabil. 2009, $1,1$.

[29] Z. Yin, X. Chen, J. L. Chen, W. L. Shen, T. M. Hieu Nguyen, L. Gao, H. W. Ouyang, Biomaterials 2010, 31, 2163.

[30] S. B. Orr, A. Chainani, K. J. Hippensteel, A. Kishan, C. Gilchrist, N. W. Garrigues, D. S. Ruch, F. Guilak, D. Little, Acta Biomater. 2015, 24, 117

[31] a) N. Barbani, G. D. Guerra, C. Cristallini, P. Urciuoli, R. Avvisati, A. Sala, E. Rosellini, J. Mater. Sci.: Mater. Med. 2012, 23, 51; b) T. T. Ruckh, D. A. Carroll, J. R. Weaver, K. C. Popat, J. Funct. Biomater. 2012, 3, 776; c) J. H. Lee, N. G. Rim, H. S. Jung, H. Shin, Macromol. Biosci. 2010, 10, 173.

[32] M. Laranjeira, R. M. A. Domingues, R. Costa-Almeida, R. L. Reis, M. E. Gomes, Small 2017, 13, 1.

[33] a) G. Abagnale, M. Steger, V. H. Nguyen, N. Hersch, A. Sechi, S. Joussen, B. Denecke, R. Merkel, B. Hoffmann, A. Dreser, U. Schnakenberg, A. Gillner, W. Wagner, Biomaterials 2015, 61, 316; b) F. Luthen, R. Lange, P. Becker, J. Rychly, U. Beck, J. G. Nebe, Biomaterials 2005, 26, 2423.

[34] a) S. Perikamana, J. Lee, T. Ahmad, Y. Jeong, D. Kim, K. Kim, H. Shin, ACS Appl. Mater. Interfaces 2015, 7, 8798; b) Z. Zheng, J. Ran, W. Chen, Y. Hu, T. Zhu, X. Chen, Z. Yin, B. C. Heng, G. Feng, H. Le, C. Tang, J. Huang, Y. Chen, Y. Zhou, P. Dominique, W. Shen, H. W. Ouyang, Acta Biomater. 2017, 51, 317; c) R. Costa-Almeida, R. M. A. Domingues, A. Fallahi, H. Avci, I. K. Yazdi, M. Akbari, R. L. Reis, A. Tamayol, M. E. Gomes, A. Khademhosseini, J. Tissue Eng. Regener. Med. 2018, 12, 1039.

[35] S.-K. Chae, E. Kang, A. Khademhosseini, S.-H. Lee, Adv. Mater. 2013, 25, 3071

[36] C. D. Han, L. Segal, J. Appl. Polym. Sci. 1970, 14, 2999.

[37] G. C. Rutledge, S. V. Fridrikh, Adv. Drug Delivery Rev. 2007, 59, 1384.

[38] G. Tetteh, A. S. Khan, R. M. Delaine-Smith, G. C. Reilly, I. U. Rehman, J. Mech. Behav. Biomed. Mater. 2014, 39, 95.

[39] C. Zhao, X. Wang, L. Gao, L. Jing, Q. Zhou, J. Chang, Acta Biomater. 2018, 73, 509 .

[40] H. H. Lu, J. Jiang, in Tissue Engineering I. Advances in Biochemical Engineering/Biotechnology, Vol. 102, Springer, Berlin, Heidelberg 2006, Ch. 138509, p. 91.

[41] a) M. Akbari, A. Tamayol, S. Bagherifard, L. Serex, P. Mostafalu, N. Faramarzi, M. H. Mohammadi, A. Khademhosseini, Adv. Healthcare Mater. 2016, 5, 751; b) A. Maziz, A. Concas, A. Khaldi, J. Stalhand, N. Persson, E. W. H. Jager, Sci. Adv. 2017, 3, e1600327.

[42] S. Thomopoulos, G. M. Genin, L. M. Galatz, J. Musculoskeletal Neuronal Interact. 2010, 10, 35.

[43] M. Benjamin, H. Toumi, J. R. Ralphs, G. Bydder, T. M. Best, S. Milz, J. Anat. 2006, 208, 471.

[44] C. T. Thorpe, H. L. Birch, P. D. Clegg, H. R. C. Screen, Int. J. Exp. Pathol. 2013, 94, 248

[45] S. Thomopoulos, J. P. Marquez, B. Weinberger, V. Birman, G. M. Genin, J. Biomech. 2006, 39, 1842.

[46] Y. Sun, F. Han, P. Zhang, Y. Zhi, J. Yang, X. Yao, H. Wang, C. Lin, X. Wen, J. Chen, P. Zhao, J. Mater. Chem. B 2016, 4, 7259.

[47] X. Li, J. Xie, J. Lipner, X. Yuan, S. Thomopoulos, Y. Xia, Nano Lett. 2009, 9, 2763

[48] J. Nowlin, M. A. Bismi, B. Delpech, P. Dumas, Y. Zhou, G. Z. Tan, Nanobiomedicine 2018, 5, 1849543518803538.

[49] I. Calejo, R. Costa-Almeida, A. I. Goncalves, D. Berdecka, R. L. Reis, M. E. Gomes, Cell Proliferation 2018, 51, e12493. 